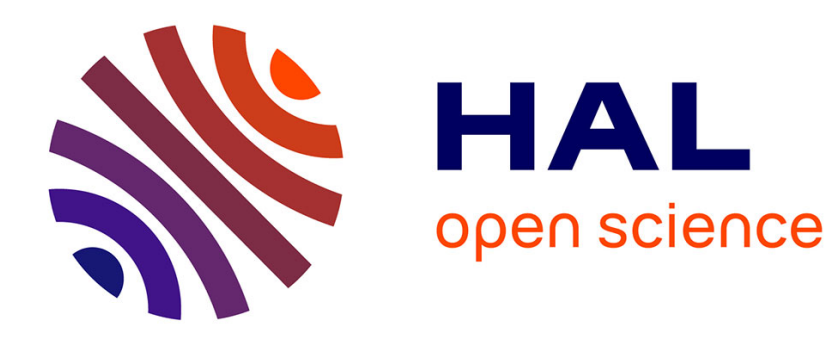

\title{
Algebraic Numbers with Elements of Small Height Haydar Göral
}

\section{To cite this version:}

Haydar Göral. Algebraic Numbers with Elements of Small Height. 2017. hal-01191860v3

\section{HAL Id: hal-01191860 https://hal.science/hal-01191860v3}

Preprint submitted on 22 Oct 2017

HAL is a multi-disciplinary open access archive for the deposit and dissemination of scientific research documents, whether they are published or not. The documents may come from teaching and research institutions in France or abroad, or from public or private research centers.
L'archive ouverte pluridisciplinaire HAL, est destinée au dépôt et à la diffusion de documents scientifiques de niveau recherche, publiés ou non, émanant des établissements d'enseignement et de recherche français ou étrangers, des laboratoires publics ou privés. 


\title{
ALGEBRAIC NUMBERS WITH ELEMENTS OF SMALL HEIGHT
}

\author{
HAYDAR GÖRAL
}

\begin{abstract}
In this paper, we study the field of algebraic numbers with a set of elements of small height treated as a predicate. We prove that such structures are not simple and have the independence property. A real algebraic integer $\alpha>1$ is called a Salem number if $\alpha$ and $1 / \alpha$ are Galois conjugate and all other Galois conjugates of $\alpha$ lie on the unit circle. It is not known whether 1 is a limit point of Salem numbers. We relate the simplicity of a certain pair with Lehmer's conjecture and obtain a model-theoretic characterization of Lehmer's conjecture for Salem numbers.
\end{abstract}

\section{INTRODUCTION}

The logarithmic height function measures the arithmetic complexity of an algebraic number and it plays a fundamental role in algebra and diophantine geometry. Before defining the logarithmic height function, we first define the Mahler measure of a polynomial over $\mathbb{C}$. For a non-zero polynomial

$$
f(x)=a_{d}\left(X-\alpha_{1}\right) \cdots\left(X-\alpha_{d}\right) \in \mathbb{C}[X],
$$

its Mahler measure is defined as the finite product

$$
m(f)=\left|a_{d}\right| \prod_{\left|\alpha_{j}\right| \geq 1}\left|\alpha_{j}\right| .
$$

By convention, the Mahler measure of zero is defined to be 1 . Let $\overline{\mathbb{Q}}$ be the field of algebraic numbers. For a non-zero $\alpha$ in $\overline{\mathbb{Q}}$ with irreducible polynomial $f(x) \in \mathbb{Z}[X]$, we define its Mahler measure as $m(\alpha)=m(f)$. The absolute non-logarithmic height of $\alpha$ is defined by

$$
H(\alpha)=m(\alpha)^{1 / d}
$$

where $d=\operatorname{deg} f$. Then the logarithmic height of $\alpha$ is defined as

$$
h(\alpha)=\log H(\alpha)=\frac{\log m(\alpha)}{d} .
$$

A central question in diophantine geometry is Lehmer's conjecture, which is still open and it states that there exists an absolute constant $c>1$ such that if $m(\alpha)>1$ then $m(\alpha) \geq c$. This question was posed by D. Lehmer [14] around 1933, who claimed that the polynomial

$$
X^{10}+X^{9}-X^{7}-X^{6}-X^{5}-X^{4}-X^{3}+X+1
$$

has the smallest Mahler measure, which is approximately 1.17628 and this is still the smallest known Mahler measure of an algebraic number. A real algebraic integer $\alpha>1$ is called a Salem number if $\alpha$ and $1 / \alpha$ are Galois conjugate and all other Galois conjugates of $\alpha$ lie on the unit circle. It is not known whether 1 is a limit point of Salem numbers. This is a special case of Lehmer's conjecture since for a Salem number $\alpha$ we have $m(\alpha)=\alpha$, and Lehmer's conjecture for Salem numbers states that 1 is not a limit point of Salem numbers. For a detailed exposition on Mahler measure and Lehmer's problem, see [20].

Classification theory is an extensive project in model theory, emerged in the work of M. Morley in the 1960's and S. Shelah in the 1970's, pursuing the categorization of first-order theories. The question of how many models a theory can have has been at the center of model

1991 Mathematics Subject Classification. 12L12, 11G50, 03C45.

Key words and phrases. height, model theory, salem numbers.

Partially supported by ValCoMo (ANR-13-BS01-0006) and MALOA (PITN-GA-2009-238381). 
theory. Stable theories are important to classify their models. If a theory is not stable then its models are difficult to handle and various to classify, and arithmetic fits in this case. A theory $T$ is said to be stable if there is no first-order formula in $T$ which has the order property. Stable theories are equipped with a notion of independence, namely the forking independence, with a well-defined set of properties, reminiscent of the algebraic independence in fields and the linear independence in vector spaces. For instance, the theory of algebraically closed fields is stable. Conversely, stability also yields some structural properties of the models and a wellknown result is that a field is $\omega$-stable if and only if it is algebraically closed [15]. A theory is simple, if the forking independence is symmetric and it is known that stable theories are simple. There are many fruitful interactions between model theory and algebra. The main examples are Ax-Grothendieck theorem via compactness [1] and Chevalley's constructibility theorem via quantifier elimination. More recently, other two important examples are the group configuration theorem by Hrushovski [12], which is a generalization of Weil's group chunk theorem, and Hrushovski's proof of the Mordell-Lang conjecture for function fields in all characteristics [13]. In this paper, we also study the interaction between model theory and diophantine geometry via the set of algebraic numbers with elements of small height. The results in this article are from the $\mathrm{PhD}$ thesis of the author [8].

The model theoretic properties of $\overline{\mathbb{Q}}$ are well understood: it is strongly minimal and has quantifier elimination in the language of rings $L_{r}=\{+,-, \cdot, 0,1\}$. In this paper, we mainly focus on the pair $\overline{\mathbb{Q}}$ together with a predicate, in particular our predicate will be the elements of height less than a given positive real number. Moreover, we study these pairs in terms of stability theory. The first motivation came from B. Zilber. In 1990, B. Zilber [24] showed that the pair

$$
(\mathbb{C}, \mu) \equiv(\overline{\mathbb{Q}}, \mu)
$$

is $\omega$-stable where $\mu$ is the group of complex roots of unity, and his pattern was based on the result of H. Mann [16]. The result of B. Zilber [24] was generalized by L. van den Dries and A. Günaydın [6] to algebraically closed fields with a multiplicative subgroup satisfying a certain diophantine condition. In this paper our predicate, namely the elements of small height, contains the group $\mu$. Moreover, the theory of $\overline{\mathbb{Q}}$ with this predicate will be a proper expansion of the theory of $(\overline{\mathbb{Q}}, \mu)$. The model theory of pairs has attracted a lot of attention recently. More generally, stable theories with a predicate were analyzed in the paper of E. Casanovas and M. Ziegler [3], where they gave criteria for a pair to be stable. Their result in [3] implies the result of B. Zilber [24] and also B. Poizat's result on the $\omega$-stability of the theory of pairs of algebraically closed fields [18].

Now we state Kronecker's theorem from diophantine geometry with no proof, as the details can be found in the book $[2,1.5 .9]$.

Theorem 1.1. (Kronecker) Let $\alpha$ in $\overline{\mathbb{Q}}$ be a non-zero algebraic number. Then $h(\alpha)=0$ if and only if it is a root of unity.

Combining Zilber's result [24] with Kronecker's theorem, we see that the pair

$$
(\overline{\mathbb{Q}},\{a \in \overline{\mathbb{Q}}: h(a)=0\})
$$

is $\omega$-stable.

Throughout this paper, the language $L_{m}$ will denote the language $\{1, \cdot\}$ where the binary operation $\cdot$ is the usual multiplication. Let $\mathbb{S}$ be the set of all Salem numbers. We put

$$
P_{b}:=\left\{a \in \overline{\mathbb{Q}}^{\times}: m(a) \leq b\right\} \text { and } \mathbb{S}_{b}=P_{b} \cap \mathbb{S}
$$

where $b \geq 1$, and we set $S_{\varepsilon}=\{a \in \overline{\mathbb{Q}}: h(a) \leq \varepsilon\}$ where $\varepsilon>0$. Both $P_{b}$ and $S_{\varepsilon}$ contain the group of roots of unity $\mu$. In other words, Lehmer's conjecture and its version for Salem 
numbers state that there exists $b>1$ such that $P_{b}=P_{1}=\mu$ and $\mathbb{S}_{b}=\mathbb{S}_{1}=\emptyset$ respectively. The pairs $\left(\overline{\mathbb{Q}}, \mathbb{S}_{b}\right)$ and $\left(\overline{\mathbb{Q}}, S_{\varepsilon}\right)$ can be seen as an $L_{r}(U)=L_{r} \cup\{U\}$ structures where $U$ is a unary relation symbol whose interpretations are $\mathbb{S}_{b}$ and $S_{\varepsilon}$ respectively. We call $S_{\varepsilon}$ the set of algebraic numbers of small height. Note that the Mahler measure of an algebraic number is again an algebraic number. Put also $S=\{a \in \overline{\mathbb{Q}}: h(a)<1\}=S_{1}$, as there is no algebraic number whose height is 1 by Lindemann's theorem.

Let $T_{\varepsilon}$ be the theory $T h\left(\overline{\mathbb{Q}}, S_{\varepsilon}\right)$. We focus on the model theory of $\left(\overline{\mathbb{Q}}, S_{\varepsilon}\right)$ in the language $L_{m}(U)=L_{m} \cup\{U\}$ and we prove a result which shows that small perturbations of the property of being a root of unity changes drastically the stability properties of the ambient structure. This means that $T_{\varepsilon}$ is model-theoretically very complicated. We also relate the simplicity of a certain pair with Lehmer's conjecture and give a model-theoretic characterization of a special case of Lehmer's conjecture. We prove the following theorem (see Theorem (3.4) and Theorem $(4.2))$.

Main Theorem. The theory of $\left(\overline{\mathbb{Q}}, S_{\varepsilon}\right)$ is not simple and has the independence property in the language $L_{m}(U)$. Moreover, the theory of $\left(\overline{\mathbb{Q}}, \mathbb{S}_{b}\right)$ is simple for some $b>1$ in $L_{m}(U)$ if and only if Lehmer's conjecture holds for all Salem numbers. Furthermore, the simplicity of the pair $\left(\overline{\mathbb{Q}}, P_{b}\right)$ for some $b>1$ in $L_{m}(U)$ implies that Lehmer's conjecture holds for all Salem numbers.

\section{Preliminaries and Height Lemmas}

2.1. The Logarithmic Height Function. Details of this subsection can be found in [2, Chapter 1] and [11, Part B, B.7].

The logarithmic height function behaves well under arithmetic operations and it satisfies the following properties:

- $h(0)=h(1)=0$,

- For a non-zero rational number $a / b$ where $a$ and $b$ are coprime,

$$
h(a / b)=\max \{\log |a|, \log |b|\},
$$

- For all $\alpha$ in $\overline{\mathbb{Q}}$, we have $h\left(\alpha^{n}\right)=n h(\alpha)$ where $n \in \mathbb{N}$,

- For all $\alpha$ and $\beta$ in $\overline{\mathbb{Q}}$, we have $h(\alpha+\beta) \leq h(\alpha)+h(\beta)+\log 2$,

- For all $\alpha$ and $\beta$ in $\overline{\mathbb{Q}}$, we have $h(\alpha \beta) \leq h(\alpha)+h(\beta)$,

- For all non-zero $\alpha$ in $\overline{\mathbb{Q}}$, we have $h(1 / \alpha)=h(\alpha)$.

2.2. Nonstandard Analysis. Next we define nonstandard extensions which will be used later on.

Definition 2.1. (Nonstandard Extension of a Structure) Let $\mathbb{M}$ be a nonempty structure in a countable language $L$. A nonstandard extension ${ }^{*} \mathbb{M}$ of $\mathbb{M}$ is an ultrapower of $\mathbb{M}$ with respect to a non-principal ultrafilter on $\mathbb{N}$.

Now let ${ }^{*} \mathbb{M}$ be a nonstandard extension of $\mathbb{M}$ with respect to a non-principal ultrafilter $D$ on $\mathbb{N}$. Note that the elements of ${ }^{*} \mathbb{M}$ are of the form $\left(x_{n}\right)_{n} / D$ where $\left(x_{n}\right)_{n}$ is a sequence in $\mathbb{M}$. We identify each element $x$ of $\mathbb{M}$ with the class of the constant sequence $(x)_{n} / D$ of * $\mathbb{M}$. In this way, the structure $\mathbb{M}$ becomes an elementary substructure of * $\mathbb{M}$. Ultraproduct of structures automatically become $\aleph_{1}$-saturated. For a subset $A$ of $\mathbb{M}$, the set ${ }^{*} A$ is defined to be the set

$$
\left\{\left(a_{n}\right)_{n} / D:\left\{n: a_{n} \in A\right\} \in D\right\} .
$$

Observe that ${ }^{*} A$ contains $A$ as mentioned above. The following sets ${ }^{*} \mathbb{N},{ }^{*} \mathbb{Z},{ }^{*} \mathbb{Q},{ }^{*} \mathbb{R}$ are called hypernatural numbers, hyperintegers, hyperrational numbers and hyperreals respectively. The elements ${ }^{*} \mathbb{R} \backslash \mathbb{R}$ are called nonstandard real numbers. Let

$$
\mathbb{R}_{\text {fin }}=\left\{x \in{ }^{*} \mathbb{R}:|x|<n \text { for some } n \in \mathbb{N}\right\}
$$


be the finite numbers. The elements in ${ }^{*} \mathbb{R} \backslash \mathbb{R}_{f i n}$ are called infinite. Note that the notion of a nonstandard extension and its properties can be extended to many-sorted structures. For more on nonstandard analysis, we direct the reader to $[7,10]$.

2.3. Height Lemmas. In this subsection, we compute heights of certain algebraic numbers which will be crucial in the proof of the Main Theorem.

Lemma 2.2. For any non-zero algebraic number $\alpha$ which is not a root of unity, the set $A(\alpha)=$ $\left\{h\left(\alpha^{q}\right): q \in \mathbb{Q}\right\}$ is dense in the positive real numbers.

Proof. Let $\alpha$ be a non-zero algebraic number which is not a root of unity. Then by Theorem (1.1), we know that $h(\alpha)>0$. Furthermore, by the properties of the logarithmic height function given in Section 2.1, we have that $h\left(\alpha^{q}\right)=|q| h(\alpha)$ for any $q \in \mathbb{Q}$. Given an interval $(a, b)$ where $0 \leq a<b$; choose $r \in \mathbb{Q}$ such that $a / h(\alpha)<r<b / h(\alpha)$. Then we conclude that $a<h\left(\alpha^{r}\right)<b$.

Lemma 2.3. Let $p_{1}, \ldots, p_{k}$ and $m_{1}, \ldots, m_{k}$ be positive natural numbers. Then

$$
h\left(p_{1}{ }^{1 / m_{1}} \cdots p_{k}{ }^{1 / m_{k}}\right)=h\left(p_{1}{ }^{1 / m_{1}}\right)+\cdots+h\left(p_{1}{ }^{1 / m_{k}}\right) .
$$

Proof. Put $m=m_{1} \cdots m_{k}$ and $n_{i}=m / m_{i}$. As before, we know that for any algebraic number $\alpha$ and rational number $q$ we have that $h\left(\alpha^{q}\right)=|q| h(\alpha)$. Therefore, we see that

$$
h\left(p_{1}{ }^{1 / m_{1}} \cdots p_{k}{ }^{1 / m_{k}}\right)=\frac{h\left(p_{1}^{n_{1}} \cdots p_{k}^{n_{k}}\right)}{m} .
$$

Since for a natural number $n \geq 1$ we have $h(n)=\log n$, we conclude that

$$
\frac{h\left(p_{1}^{n_{1}} \cdots p_{k}^{n_{k}}\right)}{m}=\frac{h\left(p_{1}^{n_{1}}\right)+\cdots+h\left(p_{k}^{n_{k}}\right)}{m}=h\left(p_{1}{ }^{1 / m_{1}}\right)+\cdots+h\left(p_{1}{ }^{1 / m_{k}}\right) .
$$

Lemma 2.4. Let $p_{1}, \ldots, p_{k}, p_{k+1}$ and $m_{1}, \ldots, m_{k}, m_{k+1}$ be natural numbers such that the product $p_{1} \cdots p_{k}$ and $p_{k+1}$ are coprime. Then we have

$$
h\left(\frac{p_{1}{ }^{1 / m_{1}} \cdots p_{k}{ }^{1 / m_{k}}}{p_{k+1}{ }^{1 / m_{k+1}}}\right)=\max \left\{h\left(p_{1}{ }^{1 / m_{1}} \cdots p_{k}{ }^{1 / m_{k}}\right), h\left(p_{k+1}{ }^{1 / m_{k+1}}\right)\right\} .
$$

Proof. Put $m=m_{1} \cdots m_{k} m_{k+1}$ and $n_{i}=m / m_{i}$. Recall that for coprime integers $a$ and $b$, we have $h(a / b)=\max \{\log |a|, \log |b|\}=\max \{h(a), h(b)\}$. Thus by the properties of the logarithmic height function in Section 2.1, we have

$$
\begin{gathered}
h\left(\frac{p_{1}{ }^{1 / m_{1}} \cdots p_{k}{ }^{1 / m_{k}}}{p_{k+1}{ }^{1 / m_{k+1}}}\right)=\frac{1}{m} h\left(\frac{p_{1}^{n_{1}} \cdots p_{k}^{n_{k}}}{p_{k+1}{ }^{n_{k+1}}}\right) \\
=\frac{1}{m} \max \left\{h\left(p_{1}^{n_{1}} \cdots p_{k}^{n_{k}}\right), h\left(p_{k+1}^{n_{k+1}}\right)\right\},
\end{gathered}
$$

and the lemma follows from the properties of the logarithmic height function again.

Recall that $\mu$ is the set of complex roots of unity. Utilizing Kronecker's theorem, we can define $\mu$ uniformly in the pair $\left(\overline{\mathbb{Q}}, S_{\varepsilon}\right)$ for any positive $\varepsilon$.

Lemma 2.5. Let $\phi(x)$ be the formula $x \neq 0 \wedge \forall y(U(y) \rightarrow U(x y))$ in the language $L_{m}(U)$. Then for any positive $\varepsilon$, the formula $\phi(x)$ defines $\mu$ in the pair $\left(\overline{\mathbb{Q}}, S_{\varepsilon}\right)$. In particular, the theory of $\left(\overline{\mathbb{Q}}, S_{\varepsilon}\right)$ expands the theory of $(\overline{\mathbb{Q}}, \mu)$.

Proof. Let $\varepsilon$ be a positive real number. A root of the unity satisfies the formula since the height function satisfies the inequality $h(x y) \leq h(x)+h(y)$ and the height of a root of unity is 0 . Now suppose $\alpha$ satisfies the formula. If we take $y=1$ in the formula, we see that $h(\alpha) \leq \varepsilon$ and so we have $\alpha \in S_{\varepsilon}$. Then letting $y=\alpha$, we get $h(\alpha) \leq \varepsilon / 2$ and thus $\alpha^{2} \in S_{\varepsilon}$. So taking powers of $\alpha$, we conclude that $h(\alpha) \leq \varepsilon / n$ for all $n \geq 1$ and hence $h(\alpha)=0$. The lemma follows from Kronecker's theorem (1.1). 


\section{Elements of Small Height}

In this section, we turn our attention to the model-theoretic properties of the field of algebraic numbers expanded by a predicate to denote elements of small height. We have all the tools to work on these pairs.

3.1. Simplicity and Independence Property. In this subsection we give two definitions for theories, namely simple theories and NIP, which play a significant role in the classification theory introduced by Shelah [19]. Then we prove the first part of the Main Theorem.

Definition 3.1. A formula $\phi(x, y)$ has the tree property (TP) if there is a tree of parameters $\left(a_{s}: \emptyset \neq s \in \omega^{<\omega}\right)$ such that

(i) For all $s \in \omega^{<\omega},\left(\phi\left(x, a_{s i}\right): i<\omega\right)$ is 2-inconsistent.

(ii) For all $\sigma \in \omega^{\omega},\left(\phi\left(x, a_{s}\right): \emptyset \neq s \subset \sigma\right)$ is consistent.

We say that $\mathrm{T}$ is simple if no formula has the tree property.

Example 3.2. The theory of dense linear orders without end points (DLO) is not simple because the formula $\varphi(x, y, z): y<x<z$ has tree property. Let $I$ be the set of rational numbers in the interval $(0,1)$ which is a model of DLO. We fix a tree of parameters $\left(q_{s}: \emptyset \neq s \in \omega^{<\omega}\right)$ in this model for the formula $\varphi$ above which we will need in order to prove the first part of our Main Theorem.

For more on simplicity we refer the reader to [23, Chapter 7].

Definition 3.3. Let $T$ be a complete $L$-theory where $L$ is a language. An $L$-formula $\phi(x, y)$ is said to have the independence property $(I P)$ if in every model $M$ of $T$ there is for each $n$ a family of tuples $b_{1}, \ldots, b_{n}$, such that for each of the $2^{n}$ subsets $I$ of $\{1, \ldots, n\}$ there is a tuple $a_{I}$ in $M$ for which

$$
M \models \phi\left(a_{I}, b_{i}\right) \Longleftrightarrow i \in I .
$$

A theory $T$ is called NIP if no formula has $I P$.

In other words, a theory $T$ has the independence property, if there is a first-order formula in some model of $T$ that can code any given subset of an arbitrarily large finite set. It is known that stable theories are NIP. Moreover $T$ is stable if and only if $T$ is simple and NIP. For more on the subject; see [19].

Now we are ready to prove the first part of our main theorem using lemmas (2.2), (2.3) and (2.4). Note that $S_{\varepsilon}$ is not a group under multiplication (or addition). In lemma (2.5), we proved that the theory of $\left(\overline{\mathbb{Q}}, S_{\varepsilon}\right)$ expands the theory of $(\overline{\mathbb{Q}}, \mu)$ which is $\omega$-stable. On the contrary, the theory of $\left(\overline{\mathbb{Q}}, S_{\varepsilon}\right)$ is very complicated.

Theorem 3.4. The theory of $\left(\overline{\mathbb{Q}}, S_{\varepsilon}\right)$ is not simple and has the independence property in the language $L_{m}(U)$.

Proof. Recall that the Mahler measure of an algebraic number is again an algebraic number. Therefore by Lindemann's theorem, the logarithmic height of an algebraic number $\alpha$ is transcendental if $h(\alpha) \neq 0$. First we prove that the theory is not simple. In order to show this, we exhibit a formula which has the tree property. Actually, the argument from Case 2 below also works in Case 1, and Case 1 is included to show the main idea of the proof in a more transparent way.

Case 1: The element $\varepsilon$ is in the range of the logarithmic height function, that is to say $\varepsilon=h(\alpha)$ for some $\alpha \in \overline{\mathbb{Q}}$. Now put

$$
\phi(x, y, z, t): U\left(\frac{t y}{x}\right) \wedge U\left(\frac{t x}{z}\right) .
$$


Observe that for any rational number $r, s \in(0,1)$,

$$
h\left(\alpha \frac{\alpha^{r}}{\alpha^{s}}\right) \leq \varepsilon \Longleftrightarrow r \leq s .
$$

Furthermore if $\left(r_{1}, s_{1}\right)$ and $\left(r_{2}, s_{2}\right)$ are disjoint intervals of $(0,1)$, where $r_{1}<s_{1}<r_{2}<s_{2} \in \mathbb{Q}$, then we cannot have

$$
\left(\overline{\mathbb{Q}}, S_{\varepsilon}\right) \models \exists x\left(\phi\left(x, \alpha^{r_{1}}, \alpha^{s_{1}}, \alpha\right) \wedge \phi\left(x, \alpha^{r_{2}}, \alpha^{s_{2}}, \alpha\right)\right),
$$

otherwise we have

$$
h\left(\alpha \frac{x}{\alpha^{s_{1}}}\right) \leq \varepsilon \quad \text { and } \quad h\left(\alpha \frac{\alpha^{r_{2}}}{x}\right) \leq \varepsilon
$$

Therefore, if we multiply these elements, we obtain that $h\left(\alpha^{2} \frac{\alpha^{r_{2}}}{\alpha^{s_{1}}}\right) \leq 2 \varepsilon$. This is a contradiction, since $s_{1}<r_{2}$ and $h\left(\alpha^{2}\right)=2 \varepsilon$. For a tuple $\beta=\left(\beta_{1}, \ldots, \beta_{m}\right) \in \mathbb{Q}^{m}$, we put $\alpha^{\beta}=\left(\alpha^{\beta_{1}}, \ldots, \alpha^{\beta_{m}}\right)$. Combining (1) and the previous argument, we see that the formula $\phi(x, y, z, t)$ has the tree property if we take the parameters $\left(\alpha^{q_{s}}, \alpha\right)$ where the parameters $\left(q_{s}: \emptyset \neq s \in \omega^{<\omega}\right)$ are as given in example (3.2).

Case 2: The element $\varepsilon$ is not in the range of the logarithmic height function. Without loss of generality, we can assume that $\varepsilon=1$ i.e $S_{\varepsilon}=S$ as 1 is not transcendental. This time we use the fact that the range of the logarithmic height function is dense in the positive reals. Again we set

$$
\phi(x, y, z, t): U\left(\frac{t y}{x}\right) \wedge U\left(\frac{t x}{z}\right) .
$$

We will show that this formula has the tree property by finding some parameters in some model of $T_{\varepsilon}$. Let ${ }^{*} \mathbb{M}$ be a nonstandard extension of the many-sorted structure

$$
\mathbb{M}=\left(\overline{\mathbb{Q}},+,-, \cdot, 0,1, h, \mathbb{R}_{\geq 0}, 2^{x},<, \mathbb{Q}\right) .
$$

Then the logarithmic height function extends to $* \overline{\mathbb{Q}}$ and it takes values in positive hyperreal numbers. We also denote this extension as $h$. Then the pair $\left({ }^{*} \overline{\mathbb{Q}},{ }^{*} S\right)$ is an elementary extension of $(\overline{\mathbb{Q}}, S)$ in $L_{m}(U)$. Note that ${ }^{*} S$ is the set of hyperalgebraic numbers whose heights are less than 1. Let $s t(a)$ denote the standard part of a finite hyperreal number. By lemma (2.2), we know that the sequence $\left\{h\left(2^{k}\right)\right\}$ is dense in positive real numbers where $k$ is a rational number. In particular, there is a hyperrational number $q$ such that $q<1 / \log 2$ and $\operatorname{st}\left(h\left(2^{q}\right)\right)=1$. In other words, $q<1 / \log 2$ and it is infinitely close to the real number $1 / \log 2$. Observe again that for any rational number $r, s \in(0,1)$,

$$
h\left(2^{q} \frac{2^{r}}{2^{s}}\right) \leq 1 \Longleftrightarrow r \leq s .
$$

Furthermore if $\left(r_{1}, s_{1}\right)$ and $\left(r_{2}, s_{2}\right)$ are disjoint intervals of $(0,1)$, where $r_{1}<s_{1}<r_{2}<s_{2} \in \mathbb{Q}$, then we cannot have

$$
\left({ }^{*} \overline{\mathbb{Q}},{ }^{*} S\right) \models \exists x\left(\phi\left(x, 2^{r_{1}}, 2^{s_{1}}, 2^{q}\right) \wedge \phi\left(x, 2^{r_{2}}, 2^{s_{2}}, 2^{q}\right)\right),
$$

otherwise we have

$$
h\left(2^{q} \frac{x}{2^{s_{1}}}\right) \leq 1 \quad \text { and } \quad h\left(2^{q} \frac{2^{r_{2}}}{x}\right) \leq 1
$$

Therefore, if we multiply these elements, we obtain that

$$
h\left(2^{2 q} \frac{2^{r_{2}}}{2^{s_{1}}}\right) \leq 2
$$

Similarly this is a contradiction, as $s_{1}<r_{2}$ and $\operatorname{st}\left(h\left(2^{2 q}\right)=2\right.$ imply

$$
h\left(2^{2 q} \frac{2^{r_{2}}}{2^{s_{1}}}\right)>2 .
$$


Thus the formula $\phi(x, y, z, t)$ has the tree property if we take the parameters $\left(2^{q_{s}}, 2^{q}\right)$ where the parameters $\left(q_{s}: \emptyset \neq s \in \omega^{<\omega}\right)$ are as given in example (3.2), and the tuple $2^{q_{s}}$ is defined as in Case 1 . Hence $T_{\varepsilon}$ is not simple.

Now we show that $T_{\varepsilon}$ has the independence property. For simplicity, we assume that $\varepsilon=1$. Let $\phi(x, y)$ be the formula

$$
U(x / y)
$$

We will show that this formula has IP. Let $n \geq 1$ be given. Let $p_{1}, \ldots, p_{n}$ be distinct prime numbers. Put $b_{m}=p_{m}^{k_{m}}$ for $m=1, \ldots, n$, where $k_{m} \in \mathbb{Q}$ will be chosen later. Now let $I$ be a subset of $\{1, \ldots, n\}$ and $r$ be the size of $I$. For $i \in I$, by lemma (2.2) we can choose $e_{i} \in \mathbb{Q}$ such that

and we set

$$
\frac{1}{r}<h\left(p_{i}{ }^{e_{i}}\right) \leq \frac{1}{r}+\frac{1}{(n+1)^{2}}
$$

$$
a_{I}=\prod_{i \in I} p_{i}^{e_{i}}
$$

By lemma (2.3), we have

$$
1<h\left(a_{I}\right) \leq 1+\frac{1}{n+1} .
$$

Now by lemma (2.2), choose $k_{m}$ such that

$$
\frac{1}{n+1}<h\left(b_{m}\right)<1 .
$$

Finally by lemma (2.4) and the properties of the height function, we get $\phi\left(a_{I}, b_{i}\right)$ if and only if $i \in I$. This yields that $\phi(x, y)$ has the independence property and hence $T_{\varepsilon}$ is not NIP.

\section{Salem Numbers and Lehmer's COnjecture}

Lehmer's conjecture (1933): There exists an absolute constant $c>1$ such that if $\alpha$ is not a root of unity nor zero, then $m(\alpha) \geq c$.

This conjecture stands still open. The best known result is due to E. Dobrowolski [5] in 1979, who proved that for $d \geq 2$,

$$
m(\alpha)>1+\frac{1}{1200} \frac{(\log \log d)^{3}}{\log d},
$$

where $d=\operatorname{deg}(\alpha)$. A polynomial $p(x)$ of degree $d$ is called reciprocal if $x^{d} p(1 / x)= \pm p(x)$. Furthermore, an algebraic number $\alpha$ is reciprocal if it is Galois conjugate to $1 / \alpha$, in other words its minimal polynomial is reciprocal. In 1971, C. Smyth [22] showed that for $\alpha$ a nonreciprocal algebraic number

$$
m(\alpha) \geq m\left(x^{3}-x-1\right)=\theta \approx 1.3247,
$$

where $\theta$ is the real root of $x^{3}-x-1$. Thus the remaining and the difficult case for Lehmer's conjecture is the set of reciprocal algebraic numbers. The next definition plays a central role in Lehmer's conjecture.

Definition 4.1. A real algebraic integer $\alpha>1$ is called a Salem number if $\alpha$ and $1 / \alpha$ are Galois conjugate and all others Galois conjugates of $\alpha$ lie on the unit circle.

So a Salem number is reciprocal. Observe that for a Salem number $\alpha$ and a positive integer $n$, the element $\alpha^{n}$ is also a Salem number, and we have

$$
m\left(\alpha^{n}\right)=m(\alpha)^{n}=\alpha^{n} .
$$


In general this is not true for an arbitrary algebraic number. It is an open question whether 1 is a limit point of Salem numbers. This is a special case of Lehmer's conjecture. The smallest known Salem number $\alpha$ was given by D. Lehmer [14] which is the root of the polynomial

$$
X^{10}+X^{9}-X^{7}-X^{6}-X^{5}-X^{4}-X^{3}+X+1
$$

with $\alpha \approx 1.17628$ and not many Salem numbers are known in the interval $(1,1.3)$. The list of small Salem numbers can be found in the link [25]. For more about Lehmer's conjecture and Salem numbers, we direct the reader to [20,21]. The rest of the paper is devoted to Lehmer's conjecture via model theory. Let $\mathbb{S}$ be the set of all Salem numbers. Recall that

$$
P_{b}:=\left\{a \in \overline{\mathbb{Q}}^{\times}: m(a) \leq b\right\} \text { and } \mathbb{S}_{b}=P_{b} \cap \mathbb{S}
$$

where $b \geq 1$. Note that $\mathbb{S}_{b}$ is the set of Salem numbers $\leq b$. Lehmer's conjecture for Salem numbers is equivalent of there exists $b>1$ such that $\mathbb{S}_{b}=\mathbb{S}_{1}=\emptyset$. The next Theorem relates the simplicity of the pair $\left(\overline{\mathbb{Q}}, \mathbb{S}_{b}\right)$ with Lehmer's conjecture and it is the other part of the Main Theorem. In particular, it states that the simplicity of the pair $\left(\overline{\mathbb{Q}}, \mathbb{S}_{b}\right)$ for some $b>1$, which is a weaker statement than $\omega$-stability, is equivalent of Lehmer's conjecture for Salem numbers.

Theorem 4.2. The theory of $\left(\overline{\mathbb{Q}}, \mathbb{S}_{b}\right)$ is simple for some $b>1$ in $L_{m}(U)$ if and only if Lehmer's conjecture holds for all Salem numbers. Moreover, the simplicity of the pair $\left(\overline{\mathbb{Q}}, P_{b}\right)$ for some $b>1$ in $L_{m}(U)$ implies that Lehmer's conjecture holds for all Salem numbers.

Proof. If Lehmer's conjecture is true for Salem numbers, then we know that the pair $\left(\overline{\mathbb{Q}}, \mathbb{S}_{b}\right)=$ $(\overline{\mathbb{Q}}, \emptyset)$ is $\omega$-stable for some $b>1$. Now suppose that $\left(\overline{\mathbb{Q}}, \mathbb{S}_{b}\right)$ is simple for some $b>1$. Assume that 1 is a limit point of Salem numbers in order to get a contradiction. Then we can choose a Salem number $\alpha$ and a positive integer $n$ such that $\alpha^{n}$ is very close to $b$. Denote the integer part of $a$ as $[a]$. Let ${ }^{*} \mathbb{M}=\left({ }^{*} \overline{\mathbb{Q}},+,-, \cdot, 0,1, m,{ }^{*} \mathbb{R}_{\geq 1},{ }^{*} \mathbb{Q},{ }^{*} \mathbb{N},<, g,[\cdot]\right)$ be a nonstandard extension of the many-sorted structure $\mathbb{M}=\left(\overline{\mathbb{Q}},+,-, \cdot, 0,1, m, \mathbb{R}_{\geq 1}, \mathbb{Q}, \mathbb{N},<, g,[\cdot]\right)$ where $g$ is the binary function from $\overline{\mathbb{Q}} \times \mathbb{N}$ to $\overline{\mathbb{Q}}$ defined as $g(a, b)=a^{b}$. The Mahler measure $m$ extends to * $\overline{\mathbb{Q}}$ and it takes values in positive hyperreal numbers $\geq 1$. We also denote this extension by $m$. Now the pair $\left(* \overline{\mathbb{Q}},{ }^{*} \mathbb{S}_{b}\right)$ is an elementary extension of $\left(\overline{\mathbb{Q}}, \mathbb{S}_{b}\right)$ in $L_{m}(U)$. Note that ${ }^{*} \mathbb{S}_{b}$ is the set of hyperalgebraic Salem numbers that are less than $b$. Then there is a nonstandard Salem number $\beta>1$ which is infinitely close to 1 , and an infinite nonstandard natural number $N$ such that $\beta^{N}<b$ and $\operatorname{st}\left(\beta^{N}\right)=b$. Put

$$
\phi(x, y, z, t): U\left(\frac{t y}{x}\right) \wedge U\left(\frac{t x}{z}\right)
$$

as before. Observe that for any rational numbers $r$ and $s$ in $(0,1)$, we have

$$
m\left(\beta^{N} \frac{\beta^{[N r]}}{\beta^{[N s]}}\right) \leq b \Longleftrightarrow r \leq s
$$

since $N$ is infinite. Similar to the proof of Theorem (3.4), the formula $\phi(x, y, z, t)$ has the tree property by taking the parameters $a_{s}=\left(\beta^{\left[N q_{s}\right]}, \beta^{N}\right)$ which are coming from example (3.2). Hence $\left(\overline{\mathbb{Q}}, \mathbb{S}_{b}\right)$ is not simple, a contradiction. The proof of the second part follows from the methodology for proving the first part of the theorem.

Remark 4.3. We call an algebraic number $\alpha$ multiplicative if $m\left(\alpha^{n}\right)=m(\alpha)^{n}$ for all natural numbers $n \geq 1$. Let $D_{b}$ be the set of multiplicative algebraic numbers whose Mahler measure is less than $b$. Note that $D_{b}$ contains $\mathbb{S}_{b}$. Then the same proof of Theorem (4.2) shows that the simplicity of the pair $\left(\overline{\mathbb{Q}}, D_{b}\right)$ for some $b>1$ in $L_{m}(U)$ is equivalent to Lehmer's conjecture for multiplicative algebraic numbers.

The following conjecture is the model-theoretic characterization of Lehmer's conjecture.

Conjecture 4.4. The theory of $\left(\overline{\mathbb{Q}}, P_{b}\right)$ is stable for some $b>1$ in $L_{m}(U)$. Moreover the stability of the pair $\left(\overline{\mathbb{Q}}, P_{b}\right)$ in $L_{m}(U)$ for some $b>1$ implies Lehmer's conjecture. 
One way of showing the simplicity is to find a notion of independence which is symmetric and satisfying the axioms of non-forking [23, Chapter 7]. In $[8,9]$, the author characterized the independence in a sufficiently saturated model $(\mathcal{U}, \mathcal{G})$ of $(\overline{\mathbb{Q}}, \mu)$ in the language $L_{r}(U)$. More precisely, let $A$ and $B$ be two algebraically closed sets in the pair $(\mathcal{U}, \mathcal{G})$. Then

$$
A \underset{A \cap B}{\stackrel{(\mathcal{U}, \mathcal{G})}{\downarrow}} B \text { if and only if } A \underset{A \cap B, \mathcal{G}}{\downarrow} B, \mathcal{G} .
$$

Hence in a sufficiently saturated model of $\left(\overline{\mathbb{Q}}, P_{b}\right)$ for a small $b$, if the theory is simple then the independence should be the independence given above.

When $1<b<1.3$, by [20] we know that every element $\alpha$ in $P_{b}$ is reciprocal. By [3, 17], every formula in the pair $\left(\overline{\mathbb{Q}}^{\times}, P_{b}\right)$ is bounded, which means every formula in the pair is equivalent to a formula of the form $Q \alpha \in P_{b} \phi(\bar{x}, \bar{\alpha})$ where $Q$ is a tuple of quantifiers and $\phi(\bar{x}, \bar{\alpha})$ is an $L_{m}$-formula. Moreover by $[3,17]$ again, if the induced structure on $P_{b}$ is stable or does not have the finite cover property, then the pair $\left(\overline{\mathbb{Q}}^{\times}, P_{b}\right)$ is stable. Hence, understanding the induced structure on $P_{b}$ and how $P_{b}$ (a set of reciprocal algebraic integers) intersects a set of the form $\left\{\left(x_{1}, \ldots, x_{n}\right): x_{1}{ }^{a_{1}} \cdots x_{n}{ }^{a_{n}}=1, x_{i} \in \overline{\mathbb{Q}}^{\times}, a_{i} \in \mathbb{Z}\right\}$ will lead to the stability of the pair, thus to Lehmer's conjecture. Another direction is first to show a weaker result that the pair $\left(\overline{\mathbb{Q}}^{\times}, P_{b}\right)$ is NIP in $L_{m}(U)$. By the paper of A. Chernikov and P. Simon [4], again we need to understand the induced structure on $P_{b}$. If the induced structure on $P_{b}$ is NIP, then so is the pair $\left(\overline{\mathbb{Q}}^{\times}, P_{b}\right)$. We end the paper by posing the following question which is also related to Lehmer's conjecture.

- Is $\mu$ definable in $\left(\overline{\mathbb{Q}}, P_{b}\right)$ for some $b>1$ ?

\section{REFERENCES}

[1] J. Ax, Injective endomorphisms of varieties and schemes, Ann. Math., 88, 239-271 (1969).

[2] E. Bombieri, W. Gubler, Heights in Diophantine Geometry, Cambridge University Press; 1 edition (September 24, 2007).

[3] E. Casanovas, M. Ziegler, Stable theories with a new predicate, J. Symbolic Logic 66 (2001) 1127-1140.

[4] A. Chernikov, P. Simon, Externally definable sets and dependent pairs, Israel J. of Math., Volume 194, Issue 1 (2013) 409-425.

[5] E. Dobrowolski, On a question of Lehmer and the number of irreducible factors of a polynomial, Acta Arith. 34 (1979), 391-401.

[6] L. van den Dries and A. Günaydın, The Fields of Real and Complex Numbers with a Small Multiplicative Group, Proc. London Math. Soc. (3) 93 (2006) 43-81.

[7] R. Goldblatt, Lectures on the Hyperreals, A Introduction to Nonstandard Analysis Springer-Verlag, New York, 1998.

[8] H. Göral, Model Theory of Fields and Heights, Ph.D thesis, Lyon, 2015.

[9] H. Göral, Tame Expansions of $\omega$-Stable Theories and Definable Groups, Notre Dame Journal of Formal Logic, to appear.

[10] C. W. Henson, Foundation of Nonstandard Analysis, A Gentle Introduction to Nonstandard Extensions, Lecture Notes.

[11] M. Hindry, J.H. Silverman, Diophantine Geometry, An Introduction, Springer-Verlag, 2000.

[12] E. Hrushovski, Contributions to stable model theory, Ph.D thesis, Berkeley, 1986.

[13] E. Hrushovski, The Mordell-Lang Conjecture for function fields, J. Amer. Math. Soc. 9 (1996), 667-690.

[14] D. H. Lehmer, Factorization of certain cyclotomic functions, Ann. of Math. (2) 34 (1933), 461-479

[15] A. Macintyre, $\omega_{1}$-categorical fields, Fundamenta Mathematicae 70, no. 3, pages 253-270, 1971.

[16] H. Mann, On linear relations between roots of unity, Mathematika 12 (1965) 107-117.

[17] A. Pillay, The model-theoretic contents of Lang's conjecture, in E. Bouscaren (ed.) Model Theory and Algebraic Geometry, Lectures Notes in Mathematics 1696 (1998), 101-106.

[18] B. Poizat, Paires de structures stables, J. Symbolic Logic, 48, 239-249, (1983).

[19] S. Shelah, Classification Theory, Studies in Logic and the Foundations of Mathematics, North Holland; 2 edition (December 20, 1990).

[20] C. Smyth, The Mahler Measure of Algebraic Numbers: A survey.

[21] C. Smyth, Seventy Years of Salem Numbers: A Survey, Bull. London Math. Soc. (2015) 47 (3): 379-395. 
[22] C. Smyth, On the product of the conjugates outside the unit circle of an algebraic integer, Bull. London Math. Soc. 3 (1971), 169-175.

[23] K. Tent, M. Ziegler, A Course in Model Theory: Lecture Notes in Logic, ASL, 2012.

[24] B. Zilber, A note on the model theory of the compex field with roots of unity, Unpublished paper, www.maths.ox.ac.ukz̃ilber, 1990.

[25] Small Salem numbers, http://www.cecm.sfu.ca/ mjm/Lehmer/lists/SalemList.html

Department of Mathematics, Koç University, Rumelifeneri Yolu, 34450, Sariyer, Istanbul, TURKEY.

E-mail address: hgoral@gmail.com 